\title{
Cytomegalovirus, Chlamydia pneumoniae, and Helicobacter pylori IgG antibodies and restenosis after stent implantation: an angiographic and intravascular ultrasound study
}

F Schiele, M K Batur, M F Seronde, N Meneveau, P Sewoke, A Bassignot, G Couetdic, F Caulfield, J-P Bassand

\begin{abstract}
Objective-To determine the impact of previous infection with cytomegalovirus, Chlamydia pneumoniae, and Helicobacter pylori on neointimal proliferation after coronary angioplasty with stent implantation.

Design-The study population was made up of 180 patients who had stent implantation in a native coronary artery with systematic angiographic and intravascular ultrasound (IVUS) follow up at six months. Quantitative coronary angiography was used to assess the late lumen loss. The mean area of neointimal tissue within the stent and the ratio of neointimal tissue to stent area were assessed from IVUS images. Previous cytomegalovirus, $C$ pneumoniae, and $H$ pylori infection was identified by IgG antibody determination.

Results-Previous cytomegalovirus infection was detected in $50 \%$ of the population, previous $C$ pneumoniae in $18 \%$, and previous $H$ pylori in $33 \%$. Mean (SD) reference diameter was 2.94 (0.48) $\mathrm{mm}$ and mean minimum lumen diameter after stent implantation was $2.45(0.42) \mathrm{mm}$. At six months, the mean late loss was $0.74(0.50) \mathrm{mm}$, the mean neointimal tissue area was 3.8 (1.7) $\mathrm{mm}^{2}$, and the average ratio of neointimal tissue area to stent area was $45(18) \%$. None of these variables of restenosis was linked to any of the three infectious agents. By multivariate analysis, lesion length was the variable best correlated with mean neointimal tissue area, the ratio of neointimal tissue to stent area, and late loss, explaining respectively $31 \%, 39 \%$, and $8 \%$ of their variability.
\end{abstract}

Conclusions-Previous infection with cytomegalovirus, $C$ pneumoniae, or $H$ pylori was not a contributing factor in the process of restenosis after stent implantation.

(Heart 2001;85:304-311)

Keywords: restenosis; stent; ultrasonics; angiography; infection

Despite vast improvements in angioplasty techniques in recent years, restenosis remains the main limiting factor of percutaneous transluminal coronary angioplasty (PTCA). The restenosis process is complex and multifactorial, involving lesion, procedure, and patient related factors. For some years now, it has also been suggested that infectious factors may be implicated. The links between atherosclerosis or coronary artery disease and three infectious agents - namely a herpesvirus (cytomegalovirus) and two Gram negative bacteria (Chlamydia pneumoniae and Helicobacter pylori)-have been studied, ${ }^{1-5}$ but so far a connection between these agents and restenosis remains unconfirmed. It has been shown that there is a link between previous cytomegalovirus infection and restenosis after atherectomy, ${ }^{6}$ but this has not been confirmed in the setting of balloon angioplasty..$^{7-9}$ Similarly, a relation between $C$ pneumoniae antibodies and balloon angioplasty has been observed, ${ }^{10}$ but again the evidence for a link with the process of restenosis remains controversial. ${ }^{911} 12$

In all these studies, restenosis was measured after balloon angioplasty or directional atherectomy, settings in which the mechanism of restenosis includes both lesion recoil and neointimal proliferation. After stent implantation, on the other hand, the restenotic process is the result of neointimal proliferation only, without recoil or stent collapse, ${ }^{13}$ and therefore may represent a better model for the identification of patient related risk factors for restenosis. ${ }^{14}$

Angiographic late lumen loss has been used to assess restenosis as a quantitative variable. ${ }^{6}{ }^{7}$ However, intravascular ultrasound (IVUS) provides cross sectional images of the artery, making it possible to measure both the lumen and the stent cross sectional areas. Moreover, when IVUS images are recorded with a motorised pullback system, the neointimal tissue within the stent can be assessed to a fine degree of accuracy, and correlates well with histomorphometry. ${ }^{15}$

Our aim in this retrospective cohort study was to determine, using angiographic and IVUS imaging, the impact of previous infection with cytomegalovirus, $C$ pneumoniae, and $H$ pylori on the amount of neointimal proliferation six months after stent implantation.

\section{Methods}

POPULATION

The study population comprised 180 patients submitted to stent implantation in a native coronary artery, with systematic angiographic and IVUS follow up in all patients at six months. 

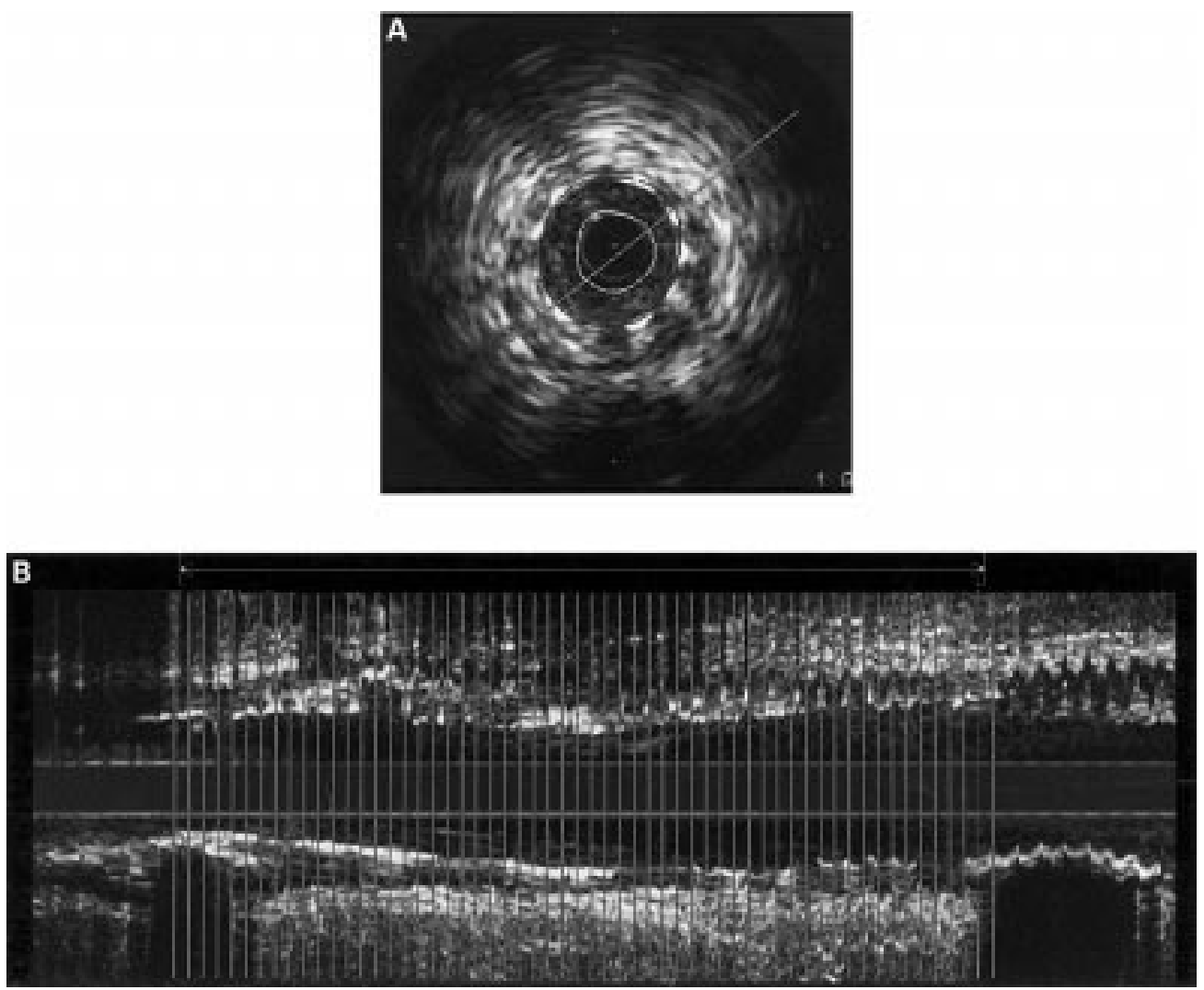

Figure 1 Example of an intravascular ultrasound image recorded six months after stent implantation. (A) Cross sectional view as used for assessment of lumen and stent area. (B) Longitudinal reconstruction as used for the selection for the level of measurements. Each line represents one manual measurement. The computer generated seven automatic measurements between each level of manual measurement, thus making a total of 57 manual measurements and 399 automatic measurements in this $12 \mathrm{~mm}$ stent.

The main reasons for the six month follow up examination were either because IVUS had been used for stent implantation (135 patients) or because of inclusion in the control arm of a clinical trial (45 patients). Additional inclusion criteria were: single stent placement (Palmaz Schatz, Johnson and Johnson; Micro Stent, Applied Vascular Engineering; or Nir, Boston Scientific Corporation) for symptomatic or demonstrable ischaemia; and informed consent for serum sample collection and systematic six month follow up. Exclusion criteria were: ostial lesion or lesion located on the left main coronary artery; vessel diameter $<3.0 \mathrm{~mm}$ by visual estimation; treatment with glycoprotein IIb/IIIa inhibitors; and unsatisfactory angioplasty result or immediate clinical complication.

ANGIOPLASTY

All procedures were performed after administration of aspirin $(500 \mathrm{mg})$ and heparin $(100 \mathrm{IU} / \mathrm{kg})$. Stents were deployed after balloon predilatation at high pressure (> $12 \mathrm{~atm})$. Overdilatation with larger balloons was carried out if necessary to achieve a satisfactory angiographic result, defined as $<20 \%$ residual stenosis. Heparin was stopped within 24 hours and patients were discharged on day 3 , on a daily dose of aspirin $250 \mathrm{mg}$ (indefinitely) and ticlopidine $500 \mathrm{mg}$ (for one month).
ANGIOGRAPHY AND QUANTITATIVE CORONARY ANALYSIS

The same two orthogonal views of the coronary artery segment submitted to stent implantation were taken before and after angioplasty, and at the six month follow up angiography. Angiograms were analysed offline using an automated edge detection algorithm (CAAS II, Pie Medical, Eindhoven, Netherlands). The minimum lumen diameter, average proximal and distal reference diameters, per cent stenosis, and lesion length were determined for each pair of orthogonal views and then averaged. The lumen loss was defined as the difference in minimum lumen diameter between stent implantation and the six month follow up, and this variable was used as an angiographic assessment of restenosis. Analysis was carried out blinded to IVUS and biological results.

ULTRASOUND PROCEDURE AND ANALYSIS

IVUS imaging was carried out using a $30 \mathrm{MHz}$ mechanical ultrasound probe and an ultrasound scanner (Cardiovascular Imaging System Inc, San Jose, California, USA). After optimisation of the machine setting and an intracoronary bolus injection of $0.5 \mathrm{mg}$ isosorbide dinitrate, images were recorded onto an S-VHS videotape recorder with an automatic $0.5 \mathrm{~cm} / \mathrm{s}$ pullback. IVUS analysis was performed off-line by a single observer, using a 
Table 1 Characteristics and risk factors of the study population $(n=180)$

\begin{tabular}{ll}
\hline Variable & \\
\hline Mean age (years) & $56(10)$ \\
Male & $158(87 \%)$ \\
& \\
Risk factors & $16(9 \%)$ \\
Diabetes & $6.0(5.8)$ \\
Mean serum glucose (mmol/l) & $99(55 \%)$ \\
Hypercholesterolaemia & $5.2(1.1)$ \\
Mean serum total cholesterol (mmol/l) & $3.2(0.9)$ \\
Mean serum LDL cholesterol (mmol/l) & $1.1(0.3)$ \\
Mean serum HDL cholesterol (mmol/l) & $135(75 \%)$ \\
Smoker & $28(16 \%)$ \\
Current smoker & $54(32 \%)$ \\
High blood pressure & $35(19 \%)$ \\
Patients with more than two risk factors & $100(90)$ \\
Mean serum creatinine ( $\mu$ mol/l) & \\
Left ventricular function & \\
Mean ejection fraction (\%) & $55(12)$ \\
Ejection fraction below $30 \%$ & $15(8 \%)$ \\
Previous myocardial infarction & $108(60 \%)$ \\
& \\
Lesion site & \\
Left anterior descending coronary artery & $80(44 \%)$ \\
Left circumflex coronary artery & $23(13 \%)$ \\
Right coronary artery & $77(43 \%)$ \\
Type of stent & \\
Palmaz-Schatz & \\
Nir & $121(67 \%)$ \\
AVE & $26(15 \%)$ \\
\hline Values are mean (SD) or n (\%). & $32(18 \%)$ \\
HDL, high density lipoprotein; LDL, low density lipoprotein.
\end{tabular}

computer assisted manual tracing system (Echo Plaque, Indec Systems Inc, Mount View, California, USA).

After longitudinal reconstruction, the segment of interest was selected (that is, the segment where the stent struts were visible). Every $0.2 \mathrm{~mm}$ along this segment, the lumen area (L-CSA) and the stent delimited area (S-CSA) were assessed by manual planimetry. Between two manual measurement levels, seven measurements were made by the computer (corresponding to 36 measurement levels/ $\mathrm{mm}$ ) and all of them were checked by the operator. An average of 600 measurements was performed within a $15 \mathrm{~mm}$ long stent, 75 manually and 525 by the computer. The method is illustrated in fig 1 . The mean lumen cross sectional area and the mean stent area were calculated. The mean neointimal tissue cross sectional area (T-CSA) within the stent was calculated as mean stent area minus mean lumen area; we also calculated the ratio of mean neointimal tissue area to mean stent area (T-CSA:S-CSA, expressed as a percentage). Mean neointimal tissue area and the T-CSA: S-CSA ratio were used as the IVUS criteria for assessment of restenosis. The reliability of the mean neointimal tissue area measurement had been assessed earlier: the intraclass correlation coefficient (ratio of interpatient variance to interpatient variance plus intraobserver variance) was 0.94 , and the standard deviation of the difference between two measurements was $0.57 \mathrm{~mm}^{2}$.

LABORATORY ANALYSES

Blood samples were collected at the six month follow up visit, centrifuged, and frozen at $-75^{\circ} \mathrm{C}$. Once all samples had been collected, they were analysed for previous cytomegalovirus, $C$ pneumoniae, or $H$ pylori infection. Cy-
Table 2 Results of biological assessment of previous cytomegalovirus, Chlamydia pneumoniae, and Helicobacter pylori infection

\begin{tabular}{lllll}
\hline Infectious agent & $\operatorname{IgG}$ & $\begin{array}{l}\operatorname{IgG}+ \\
\operatorname{IgM}\end{array}$ & $\begin{array}{l}\operatorname{IgM} \\
\text { alone }\end{array}$ & $\begin{array}{l}\operatorname{IgG}+ \\
\operatorname{IgA}\end{array}$ \\
\hline Cytomegalovirus & $91(51 \%)$ & 2 & 1 & \\
C pneumoniae & $33(18 \%)$ & & & 1 \\
H pylori & $61(33 \%)$ & & & \\
\hline
\end{tabular}

tomegalovirus antibodies (IgG and $\operatorname{IgM}$ ) were determined (Biotest, Dreieich, Germany, recombinant HCMV IgG, and enzyme linked immunosorbent assay (ELISA) for IgM), and the threshold value for positivity was set at 0.321 (IgG) and 0.523 (IgM). C pneumoniae IgG and IgA and H pylori IgG were also assessed by ELISA (Pyloriset EIA, Orion Diagnostica, Espoo, Finland and ServiMif Chlamydia, Servibio, Meudon, France, respectively), with a threshold value of 512 ( $C$ pneumoniae IgG), 40 (C pneumoniae IgA), and 300 (H pylori IgG). The analyses were performed blinded to the angiographic or IVUS results.

\section{STATISTICS}

Continuous data are presented as mean (SD) and qualitative data as percentages. Comparisons between the groups for qualitative data were made using Fisher's exact probability test or the likelihood ratio $\chi^{2}$ test, while those for quantitative data were made using the Student $t$ test or the Mann-Whitney $U$ test where appropriate. Analysis of variance (ANOVA) and regression analysis were used to determine univariate and multivariate factors related to the assessment of restenosis. Pearson's correlation coefficient $\left(r\right.$ and $r^{2}$ ) were calculated from single or multiple regression. A probability value of $\mathrm{p}<0.05$ was considered significant. The statistical analyses were performed with BMDP Dynamic statistical software (University of California, Berkeley, California, USA).

\section{Results}

POPULATION

One hundred and eighty patients had successful angioplasty, complete angiographic and IVUS follow up, and blood sample collection. The characteristics of the population are presented in table 1 . The mean (SD) age of the population was 56 (10) years; 16 (9\%) had diabetes, 99 (55\%) had hypercholesterolaemia, and $135(75 \%)$ were smokers. Thirty five $(19 \%)$ had more than two risk factors. The lesion site was the left anterior descending coronary artery in $80(44 \%)$, the left circumflex artery in $26(15 \%)$, and the right coronary artery in $77(43 \%)$.

ANGIOGRAPHIC RESULTS

The mean reference diameter was 2.94 $(0.48) \mathrm{mm}$ (range $2.2-4.6 \mathrm{~mm}$ ) and the average lesion length was 15 (11) $\mathrm{mm}$ (range 2-42 mm). The minimum lumen diameter increased from $0.99 \quad(0.35) \mathrm{mm}$ (range $0-2.27 \mathrm{~mm}$ ) before angioplasty to 2.45 (0.42) $\mathrm{mm}$ (range $1.7-3.6 \mathrm{~mm}$ ) after stent implantation, and the diameter stenosis decreased from 72 (15)\% (range $48-100 \%$ ) to 15 (8) $\%(0-40 \%)$. At the six month follow up, the 
Table 3 Angiographic and intravascular ultrasound results in relation to previous cytomegalovirus, $C$ pneumoniae, and $H$ pylori infection

\begin{tabular}{|c|c|c|c|c|c|c|}
\hline & \multicolumn{6}{|l|}{ Serostatus } \\
\hline & \multicolumn{2}{|c|}{ Cytomegalovirus } & \multicolumn{2}{|c|}{$C$ pneumoniae } & \multicolumn{2}{|l|}{ H pylori } \\
\hline & $+(n=91)$ & $-(n=89)$ & $+(n=33)$ & $-(n=147)$ & $+(n=61)$ & $-(n=119)$ \\
\hline Reference diameter (mm) & \multirow{2}{*}{\multicolumn{2}{|c|}{0.06}} & $2.93(0.49)$ & $2.94(0.48)$ & $2.96(0.47)$ & $2.93(0.48)$ \\
\hline $\mathrm{p}$ Value & & & \multicolumn{2}{|c|}{0.88} & \multicolumn{2}{|c|}{0.78} \\
\hline MLD pre-angioplasty (mm) & $1.03(0.36)$ & $0.95(0.34)$ & $1.01(0.35)$ & $0.99(0.35)$ & $0.97(0.37)$ & $1.01(0.34)$ \\
\hline $\mathrm{p}$ Value & \multicolumn{2}{|c|}{0.15} & \multicolumn{2}{|c|}{0.72} & \multicolumn{2}{|c|}{0.45} \\
\hline MLD post-angioplasty (mm) & $2.51(0.45)$ & $2.39(0.37)$ & $2.47(0.40)$ & $2.45(0.40)$ & $2.50(0.72)$ & $2.42(0.43)$ \\
\hline p Value & \multicolumn{2}{|c|}{0.06} & \multicolumn{2}{|c|}{0.77} & \multicolumn{2}{|c|}{0.24} \\
\hline MLD at follow up (mm) & $1.78(0.51)$ & $1.62(0.60)$ & $1.77(0.51)$ & $1.69(0.57)$ & $1.72(0.54)$ & $1.69(0.57)$ \\
\hline $\mathrm{p}$ Value & \multicolumn{2}{|c|}{0.05} & \multicolumn{2}{|c|}{0.44} & \multicolumn{2}{|c|}{0.71} \\
\hline Early gain $(\mathrm{mm})$ & $1.49(0.44)$ & $1.43(0.46)$ & $1.46(0.53)$ & $1.46(0.43)$ & $1.53(0.48)$ & $1.42(0.40)$ \\
\hline p Value & & & \multicolumn{2}{|c|}{0.13} \\
\hline Late loss (mm) & $0.73(0.49)$ & 0.54 & \multicolumn{2}{|c|}{$\begin{array}{ll} & 0.98 \\
0.71(0.56) & 0.77(0.50)\end{array}$} & $0.80(0.54)$ & $0.74(0.45)$ \\
\hline p Value & \multicolumn{2}{|c|}{0.51} & \multicolumn{2}{|c|}{0.59} & \multicolumn{2}{|c|}{0.62} \\
\hline Loss index $(\%)$ & $50(37)$ & $59(44)$ & $50(44)$ & $56(40)$ & $54(42)$ & $54(37)$ \\
\hline $\mathrm{p}$ Value & \multicolumn{2}{|r|}{0.13} & \multicolumn{2}{|r|}{0.48} & \multicolumn{2}{|c|}{0.92} \\
\hline Binary restenosis & $15(16.5 \%)$ & $26(32 \%)$ & $4(9 \%)$ & $37(25 \%)$ & $13(21 \%)$ & $28(24 \%)$ \\
\hline $\mathrm{p}$ Value & \multicolumn{2}{|c|}{0.047} & \multicolumn{2}{|r|}{0.08} & \multicolumn{2}{|c|}{0.73} \\
\hline Mean L-CSA (mm) & $4.7(2.6)$ & $4.7(2.4)$ & $4.8(2.4)$ & $4.7(2.4)$ & $4.6(2.2)$ & $4.7(1.9)$ \\
\hline $\mathrm{p}$ Value & \multicolumn{2}{|r|}{0.85} & & 0.83 & & 76 \\
\hline Mean S-CSA (mm) & $8.5(1.9)$ & $8.4(2.1)$ & $8.5(2.4)$ & $8.4(2.2)$ & $8.4(2.0)$ & $8.4(1.8)$ \\
\hline $\mathrm{p}$ Value & & 0.72 & & 0.83 & & 96 \\
\hline Mean T-CSA $(\mathrm{mm})$ & $4.0(1.8)$ & $3.7(1.7)$ & $3.8(1.7)$ & $3.8(1.8)$ & $3.8(1.8)$ & $3.8(1.7)$ \\
\hline $\mathrm{p}$ Value & & 0.23 & & 0.95 & & 89 \\
\hline T-CSA:S-CSA (\%) & $0.46(0.18)$ & $0.44(0.19)$ & $0.46(0.19)$ & $0.45(0.18)$ & $0.45(0.19)$ & $0.46(0.18)$ \\
\hline $\mathrm{p}$ Value & & $0.43^{\star}$ & & $0.91^{\star}$ & & $64^{\star}$ \\
\hline
\end{tabular}

Values are mean (SD)

${ }^{\star} \mathrm{p}$ values from Mann-Whitney U test.

L-CSA, lumen cross sectional area; MLD, angiographic minimum lumen diameter; S-CSA, stent cross sectional area; T-CSA, neointimal tissue cross sectional area within the stent.

minimum lumen diameter was $1.70(0.56) \mathrm{mm}$ (range $0.45-3.05 \mathrm{~mm}$ ), corresponding to an average diameter stenosis of 37 (16)\% (range $0-90 \%)$. The binary restenosis rate was $23 \%$.

IVUS RESULTS

At six months, IVUS images suitable for analysis were available for 179 patients. The average number of measured slices was 456 (39) per patient (mean length $11.4(1) \mathrm{mm}$ ). The length of the segment measured corresponded to $72 \%$ of the actual stent length. The mean neointimal tissue cross sectional area was $3.8(1.7) \mathrm{mm}^{2}$, calculated from a mean lumen area of 4.6 (2.0) $\mathrm{mm}^{2}$ and a mean stent area of 8.5 (2.0) $\mathrm{mm}^{2}$; the average T-CSA:S-CSA ratio was $45(18) \%$.

LABORATORY RESULTS

Table 2 summarises the antibody detection results. Cytomegalovirus IgG assessment was positive in 91 patients $(51 \%)$, and cytomegalovirus $\operatorname{Ig} M$ in two. In another patient, high titres

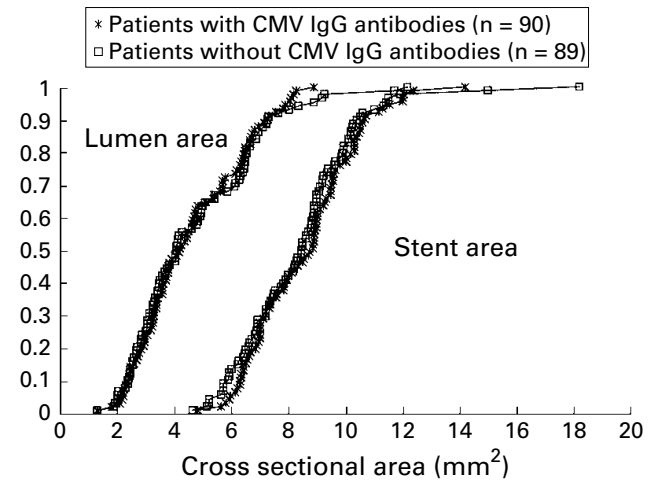

Figure 2 Cumulative curves of mean lumen and mean stent cross sectional areas in patients with and without cytomegalovirus $\operatorname{Ig} G$ antibodies. of cytomegalovirus $\operatorname{IgM}$ were detected but without significant cytomegalovirus IgG. This case was considered to be a recent and asymptomatic infection, confirmed by a further increase in cytomegalovirus IgG antibodies. $C$ pneumoniae IgG was detected in 33 patients $(18 \%)$, and in one patient both IgG and IgA were present, suggesting a recent infection. $H$ pylori IgG was detected in 61 patients $(33 \%)$.

LINKS BETWEEN RESTENOSIS AND

CYTOMEGALOVIRUS, C PNEUMONIAE, AND $H$ PYLORI SEROSTATUS

Table 3 shows the angiographic and IVUS results according to serostatus. There was a significant difference in reference diameter between patients with and without previous cytomegalovirus infection, but no difference in lesion site. Thus patients with previous cytomegalovirus infection $(\mathrm{n}=91)$ tended to have larger arteries (3.01 (0.49) $\mathrm{mm} v 2.87$ (0.43) $\mathrm{mm}, \mathrm{p}=0.06)$, a larger post-stent

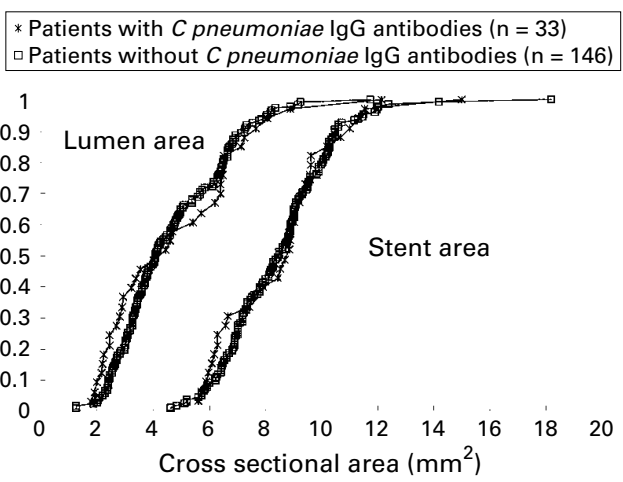

Figure 3 Cumulative curves of mean lumen and mean stent cross sectional areas in patients with and without $C$ pneumoniae IgG antibodies. 


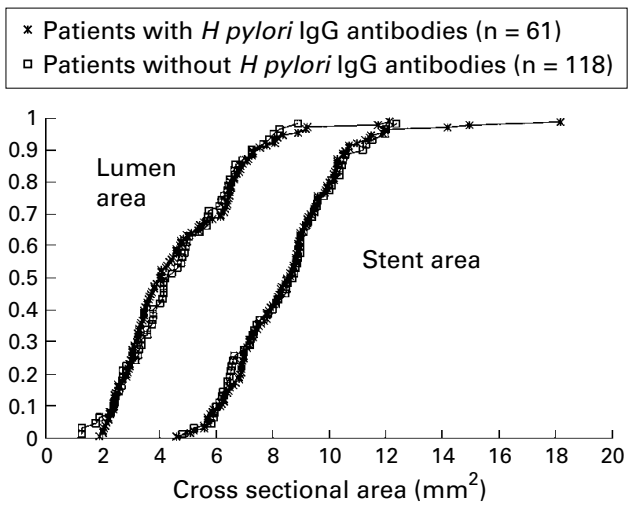

Figure 4 Cumulative curves of mean lumen and mean stent cross sectional areas in patients with and without $H$ pylori IgG antibodies.

minimum lumen diameter $(2.51(0.45) \mathrm{mm} v$ $2.39(0.37) \mathrm{mm}, \mathrm{p}=0.06)$, and a larger minimum lumen diameter at follow up (1.78 (0.51) $\mathrm{mm} v 1.62(0.60) \mathrm{mm}, \mathrm{p}=0.05)$. The restenosis rate was lower in the group with previous cytomegalovirus infection $(16.5 \%$ v $32 \%, \mathrm{p}=0.045)$, although the late loss was similar in the two groups $(0.73(0.49) \mathrm{mm} v$ $0.78(0.52) \mathrm{mm}, \mathrm{p}=0.51)$. IVUS analysis showed that the mean stent area and the mean lumen area were comparable in both groups and that there was no difference in mean neointimal tissue area $\left(4.0(1.8) \mathrm{mm}^{2}\right.$ in the group with cytomegalovirus antibodies $v 3.7$ (1.7) $\mathrm{mm}^{2}$ in the group without, $\mathrm{p}=0.24$ ). The T-CSA:S-TCSA ratio was also comparable in the two groups (44 (19)\% v $46(1.8) \%$, respectively; $\mathrm{p}=0.43$ ).

There was no difference in baseline data between the group of patients with previous $C$ pneumoniae infection and those without. At follow up, both groups had a comparable mean neointimal tissue area $\left(3.8(1.7) \mathrm{mm}^{2} v 3.8\right.$ (1.8) $\mathrm{mm}^{2}$ respectively; $\left.\mathrm{p}=0.95\right)$, T-CSA:SCSA ratio (45 (18)\% v 46 (19)\%, respectively; $\mathrm{p}=0.91)$, and angiographic late loss $(0.71$ $(0.56) \mathrm{mm} v 0.77(0.50) \mathrm{mm}$, respectively, $\mathrm{p}=0.59$ ). Similarly, the angiographic and IVUS results were comparable between the groups of patients with and without previous $H$ pylori infection. Figures 2, 3, and 4 present the cumulative curves of mean neointimal tissue area in relation to cytomegalovirus, $C$ pneumoniae, and H pylori serology. The impact of the three infectious agents and their interaction on restenosis was assessed using analysis of variance. No subgroup showed any

Table 4 Impact of previous cytomegalovirus, $C$ pneumoniae, and $H$ pylori infection on neointimal tissue area (absolute value and percentage) and on angiographic late loss (results of analysis of variance)

\begin{tabular}{lrlll}
\hline $\begin{array}{l}\text { Serostatus positive (IgG }> \\
\text { threshold) }\end{array}$ & $n$ & $\begin{array}{l}\text { Mean T-CSA } \\
\left(\mathrm{mm}^{2}\right)\end{array}$ & T-CSA:S-CSA (\%) & Late loss (mm) \\
\hline None & 58 & $3.86(1.76)$ & $46(19)$ & $0.78(0.54)$ \\
CMV & 44 & $3.91(1.77)$ & $45(18)$ & $0.74(0.52)$ \\
CP & 8 & $3.88(1.65)$ & $45(23)$ & $0.50(0.61)$ \\
HP & 17 & $2.98(1.35)$ & $37(19)$ & $0.80(0.37)$ \\
CMV + CP & 9 & $3.43(1.59)$ & $45(20)$ & $0.71(0.52)$ \\
CMV + HP & 28 & $4.32(2.01)$ & $49(18)$ & $0.74(0.45)$ \\
CP + HP & 6 & $3.70(1.53)$ & $44(19)$ & $1.09(0.49)$ \\
CMV + CP + HP & 10 & $4.00(1.92)$ & $47(20)$ & $0.64(0.56)$ \\
\hline
\end{tabular}

Values are mean (SD).

CMV, cytomegalovirus; CP, C pneumoniae; HP, H pylori; IgG, immunoglobulin G.
* Patients with CMV IgG antibodies $(\mathrm{n}=90)$; correlation coefficient $=0.48, r^{2}=0.23, \mathrm{p}<0.001$

- Patients without CMV IgG antibodies ( $n=89)$; correlation coefficient $=0.63, r^{2}=0.40, p<0.001$

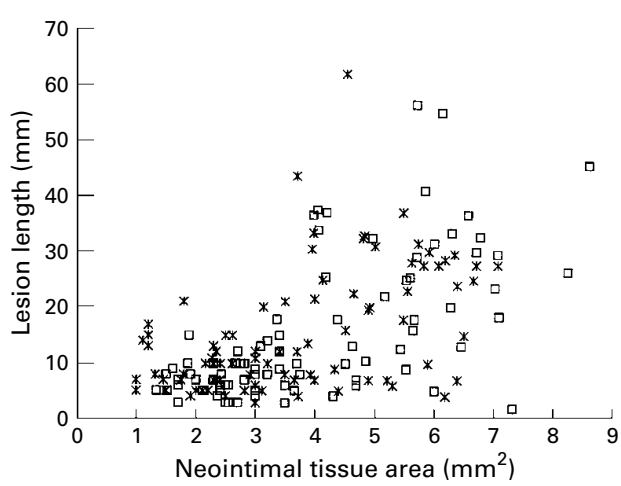

Figure 5 Plot of mean neointimal tissue area versus angiographic lesion length in patients with and without cytomegalovirus antibodies. Whole population $(n=179)$ : correlation coefficient $=0.56, r^{2}=0.31, p<0.001(T-C S A$ $=2.56+0.08$ lesion length).

\section{* Patients with $C$ pneumoniae lgG antibodies $(\mathrm{n}=33$ ); correlation coefficient $=0.67, \mathrm{p}<0.001$ \\ - Patients without $C$ pneumoniae IgG antibodies $(n=146)$; correlation coefficient $=0.56, p<0.001$}

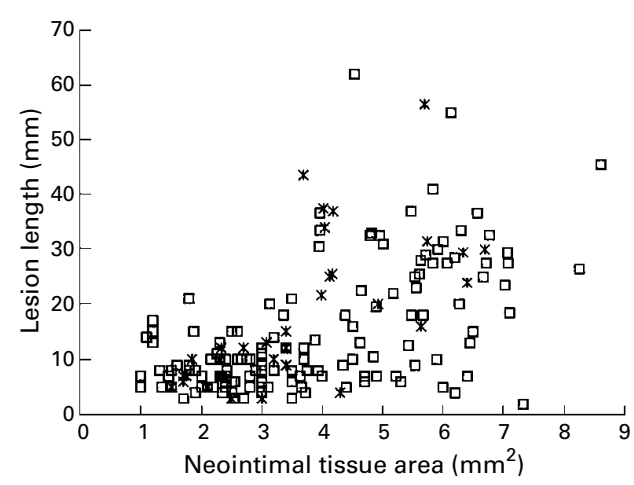

Figure 6 Plot of mean neointimal tissue area versus angiographic lesion length in patients with and without $C$ pneumoniae antibodies. Whole population $(n=179)$ : correlation coefficient $=0.56, p<0.001(T-C S A=0.61+$ 3.8 lesion length)

significant difference in mean neointimal tissue area, T-CSA:S-CSA ratio, or late loss (table 4).

By univariate analysis, the type of stent, the angiographic lesion length, and the minimum lumen diameter post-stent were found to be related to neointimal tissue area and to the T-CSA:S-CSA ratio (table 5). These variables, plus the cytomegalovirus, $C$ pneumoniae, and $H$ pylori serostatus, were entered into a stepwise multivariate regression analysis (table 6). The variables remaining in the regression model on neointimal tissue area and on the T-CSA:S-CSA ratio were the angiographic lesion length and the minimum lumen diameter post-stent implantation (multiple correlation coefficients, respectively, $r=0.59$, $r^{2}=0.35, \mathrm{p}<0.001$; and $r=0.62, r^{2}=0.39$, $\mathrm{p}<0.001)$. The variables remaining in the regression model on late loss were the lesion length and the type of stent (multiple correlation coefficient $\left.r=0.34, r^{2}=0.11, \mathrm{p}<0.001\right)$. 
* Patients with $H$ pylori lgG antibodies $(\mathrm{n}=60)$; correlation coefficient $=0.62, p<0.001$

- Patients without $H$ pylori $\lg G$ antibodies $(\mathrm{n}=119)$; correlation coefficient $=0.54, p<0.001$

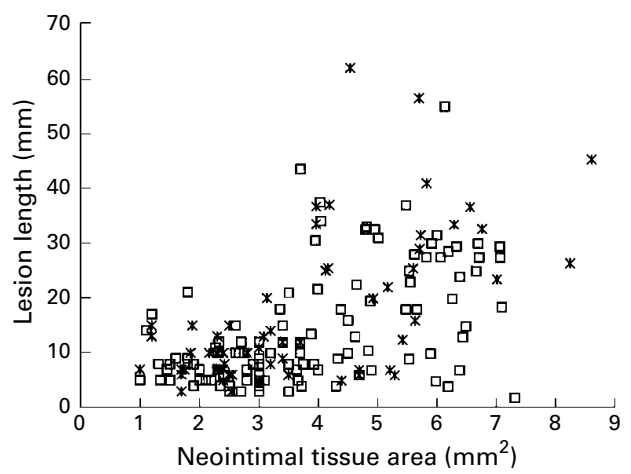

Figure 7 Plot of mean neointimal tissue area versus angiographic lesion length in patients with and without $H$ pylori antibodies. Whole population $(n=179)$ : correlation coefficient $=0.56, p<0.001(T-C S A=0.61+3.8$ lesion length)

Previous cytomegalovirus, $C$ pneumoniae, and $H$ pylori infection did not alter the linear correlation between the lesion length and the mean neointimal tissue area (figs 5, 6, and 7).

\section{Discussion}

The results of this study show that previous infection with cytomegalovirus, $C$ pneumoniae, or $H$ pylori - as ascertained by the significant presence of specific IgG antibodies-had no influence on neointimal tissue proliferation after stent implantation.

\section{STUDY DESIGN}

In comparison with previous studies, this cohort study had three specific points of design: the assessment of three infectious agents, the assessment of restenosis after stent

Table 5 Variables in relation to mean neointimal tissue area, ratio of tissue to stent area, angiographic late loss, and angiographic minimum lumen diameter at the six month follow up, by univariate analysis

\begin{tabular}{llll}
\hline Variable & Mean T-CSA & $\begin{array}{l}\text { Mean T-CSA: } \\
\text { mean S-CSA }\end{array}$ & $\begin{array}{l}\text { Angiographic late } \\
\text { loss }\end{array}$ \\
\hline Type of stent & $\mathrm{p}<0.01^{\star}$ & $\mathrm{p}<0.01^{\star}$ & $\mathrm{p}=0.01^{\star}$ \\
$\quad$ Palmaz-Schatz $(\mathrm{n}=121)$ & $4.2(1.9) \mathrm{mm}^{2}$ & $50(19) \%$ & $0.67(0.47) \mathrm{mm}$ \\
$\quad$ AVE $(\mathrm{n}=32)$ & $2.95(1.1) \mathrm{mm}^{2}$ & $34(11) \%$ & $0.91(0.55) \mathrm{mm}$ \\
$\quad$ Nir (26) & $3.1(1.2) \mathrm{mm}^{2}$ & $35(11) \%$ & $0.95(0.55) \mathrm{mm}$ \\
Angiographic lesion length & $r=0.56$ & $r=0.62$ & $r=0.20$ \\
& $\mathrm{p}<0.01^{\star \star}$ & $\mathrm{p}<0.01^{\star \star}$ & $\mathrm{p}=0.007^{\star \star}$ \\
Angiographic MLD post-stent & $r=0.02$ & $r=0.28$ & $r=0.27$ \\
& $\mathrm{p}=0.77^{\star \star}$ & $\mathrm{p}<0.01^{\star \star}$ & $\mathrm{p}<0.01^{\star \star}$ \\
\hline
\end{tabular}

Values area mean (SD).

${ }^{\star}$ Results from analysis of variance; ${ }^{\star \star} r=$ Pearson correlation coefficient, results from linear regression.

Table 6 Results of stepwise multiple regression on mean neointimal tissue cross sectional area, ratio of neointimal tissue to stent area, and angiographic late loss

\begin{tabular}{lllllc}
\hline Dependent variable & Parameter & $r$ & $r^{2}$ & ${\text { Changes in } r^{2}}^{p}$ & Value \\
\hline Mean T-CSA & Lesion length & 0.57 & 0.31 & 0.31 & $<0.001$ \\
& MLD post-stent & 0.59 & 0.33 & 0.02 & 0.08 \\
T-CSA:S-CSA & Lesion length & 0.62 & 0.039 & 0.039 & $<0.001$ \\
Late loss & MLD post-stent & 0.28 & 0.08 & 0.08 & 0.05 \\
& Type of stent & 0.33 & 0.11 & 0.03 & 0.04 \\
\hline
\end{tabular}

implantation, and the assessment by means of angiography and IVUS imaging.

\section{Infectious agents}

The relation between infection and atherosclerosis is based on the inhibition of the p53 tumour suppressor gene by immediate early antigen IE84, which favours excessive smooth muscle cell proliferation, ${ }^{16}{ }^{17}$ and similar mechanisms may explain the role of infectious agents in restenosis. It has been suggested that the arterial wall injury resulting from invasive procedures such as balloon angioplasty or atherectomy may reactivate previous infections latently harboured in stenotic lesions, thereby predisposing to restenosis. ${ }^{18}$ Furthermore, as all three infectious agents-cytomegalovirus, $C$ pneumoniae, and $H$ pylori - induce similar direct and indirect effects, it made sense to assess their impact in the setting of restenosis. ${ }^{19}{ }^{20}$ Only one study has reported the association between all three agents and coronary heart disease, ${ }^{21}$ and our report is the first to examine the impact of these three agents on restenosis.

\section{Restenosis after stent implantation}

After directional atherectomy and balloon angioplasty, late lumen loss is primarily the result of remodelling rather than neointimal formation. ${ }^{20-22}$ Conversely, after stent implantation, restenosis is the result of neointimal growth within the stent only, without arterial remodelling or stent recoil. ${ }^{13}$ Therefore stent implantation would appear to be a better model for assessing the impact of infectious agents.

\section{Assessment of restenosis}

Restenosis was assessed by two independent measurements: quantitative coronary angiography and intravascular ultrasound. IVUS assessment of the amount of neointimal proliferation after stent implantation, as quantitative data, has already been validated and correlates strongly with histomorphometry. ${ }^{15}$ The use of the mean neointimal tissue area instead of volume allows the assessment of various stent lengths and avoids the need for additional calculations such as Simpson's rule. A previous study has shown that at follow up, intimal thickness was independent of stent size, but not area. ${ }^{23}$ Therefore in order to adjust the results to the stent size, the T-CSA:S-CSA ratio was used. Previous IVUS studies have used a different method whereby 15 slices were assessed along a $15 \mathrm{~mm}$ long stent - that is, one analysis per millimetre. ${ }^{1523}$ In our study, an average of 456 slices was analysed, with one manual and 35 automatic (computed) planimetries performed every $0.2 \mathrm{~mm}$. A similar IVUS analysis method, measuring a set of 200 IVUS images by automated processing, has been used to assess the mechanism of restenosis after directional atherectomy. ${ }^{24}$

\section{BIOLOGICAL RESULTS}

No cytomegalovirus IgM antibodies were detected in any of the patients except one, suggesting the absence of acute infection. Previous 
exposure to infectious agents was considered to be a binary variable, with a titre of specific IgG antibodies above or below the threshold determined by the test manufacturers. Antibody titres were not considered to be a quantitative variable, as in previous studies, ${ }^{68}$ as no variation in a negative titre is meaningful. ${ }^{25}$ In our population, half the patients presented with cytomegalovirus IgG antibodies, almost one fifth with $C$ pneumoniae IgG, and one third with $H$ pylori IgG. This level of seroprevalence in a population with an average age of 56 years is comparable to that reported in previous studies with regard to cytomegalovirus infection, ${ }^{5-9}$ but lower than that reported for $C$ pneumoniae and $H$ pylori infection. ${ }^{5} 911$

CYTOMEGALOVIRUS ANTIBODIES AND

ANGIOGRAPHIC RESULTS

We observed a near significant trend towards larger arteries in the group with previous cytomegalovirus infection (reference diameter $3.01(0.49) \mathrm{mm} v 2.87(0.43) \mathrm{mm}, \mathrm{p}=0.06)$. This difference explained the larger minimum lumen diameter post-stent implantation and at the six month follow up observed in our study (1.78 (0.51) mm v $1.62(0.60) \mathrm{mm}, \mathrm{p}=0.05)$. Such a difference in reference diameter and minimum lumen diameter has previously been reported by Zhou and colleagues, ${ }^{6}$ in whose study the group presenting with anticytomegalovirus IgG antibodies had a trend towards a larger reference diameter $(3.23(0.42) \mathrm{mm} v$ $3.05(0.48) \mathrm{mm}, \mathrm{p}=0.07)$, and a larger minimum lumen diameter both before angioplasty $(1.29(0.44) \mathrm{mm}$ v $1.09(0.33) \mathrm{mm}$, $\mathrm{p}=0.045)$ and after angioplasty (3.18 $(0.51) \mathrm{mm}$ v $2.89 \quad(0.45) \mathrm{mm}, \mathrm{p}=0.01)$. Although observed in two independent studies, this could be a chance finding, or alternatively it could reflect a particular mechanism of development of atherosclerotic lesions in patients with previous cytomegalovirus infection.

One surprising result of the angiographic analysis was the significant difference in binary restenosis rate between patients with and without cytomegalovirus antibodies, suggesting that previous cytomegalovirus infection may have a protective effect. This finding only related to vessel size and minimum lumen diameter after stent implantation, and there was no difference in late loss in the group with cytomegalovirus antibodies. As minimum lumen diameter after stent implantation is a principal predictor of binary restenosis, ${ }^{26}$ the difference in restenosis rate can be explained by the difference in minimum lumen diameter after stent implantation, and therefore by the initial difference in vessel size.

RESTENOSIS AND PREVIOUS CYTOMEGALOVIRUS, $C$ PNEUMONIAE, AND H PYLORI INFECTION

By univariate analysis, the angiographic and IVUS measurements of restenosis were not linked to cytomegalovirus, $C$ pneumoniae, or $H$ pylori serostatus. The hypothesis of a possible interaction between two or three infectious agents and the occurrence of restenosis was not confirmed by our results. By univariate analysis, the mean neointimal tissue area, the
T-CSA:S-CSA ratio, and angiographic late loss were all related to the type of stent (particularly the Palmaz-Schatz stent), to the minimum lumen diameter after stent implantation (more late loss with a larger minimum lumen diameter post-stent), and to lesion length (the longer the lesion, the greater the mean neointimal tissue area, T-CSA:S-CSA ratio, and late loss). These variables have already been described as predictors of binary restenosis after stenting. ${ }^{27}$ By multivariate analysis, lesion length was the best correlated variable, with mean neointimal tissue area, T-CSA:S-CSA ratio, and late loss explaining, respectively, $31 \%, 39 \%$, and $8 \%$ of their variability. These correlations were not significantly altered by the presence or absence of previous cytomegalovirus, $C$ pneumoniae, or $H$ pylori infection, confirming that these infectious agents do not contribute to the restenotic process after stent implantation.

Our results are in agreement with another study after balloon angioplasty, ${ }^{79}$ but contrast with those observed after directional atherectomy. ${ }^{6}$ One possible explanation for this discrepancy is the varying degree of vascular injury caused by each of the revascularisation techniques. After atherectomy, arterial wall injury may be considerably greater than after balloon angioplasty or stent implantation, and this is reflected by the difference in early lumen gain: $1.85(0.55) \mathrm{mm}$ after atherectomy, ${ }^{6}$ $0.8 \mathrm{~mm}$ after balloon angioplasty, ${ }^{7}$ and 1.46 $(0.44) \mathrm{mm}$ in our study after stent implantation. Moreover, discrepancies between the results obtained in our study and those obtained in other studies - no matter what the angioplasty technique employed-could also be explained by differences in the assessment of infection (antibodies recorded as a quantitative or qualitative variable), the choice of end point (restenosis or clinical outcome), and the method of assessment (angiographic only or angiographic plus IVUS). ${ }^{68}$

CONCLUSIONS

The results of this study show that previous infection with cytomegalovirus, $C$ pneumoniae, or $H$ pylori was not a contributing factor in the process of restenosis after stent implantation. Therefore our data do not support the concept of identifying patients at risk by antibody detection, or of giving specific anti-infection treatment to prevent restenosis after stent implantation.

1 Melnick JL, Adam E, DeBackey ME. Cytomegalovirus and atherosclerosis. Eur Heart f 1993;14:30-8.

2 Davidson M, Kuo CC, Middaugh JP, et al. Confirmed previous infection with chlamydia pneumonia (TWAR) and its presence in early coronary atherosclerosis. Circulation 1998;98:628-33.

3 Quinn M, Foley J, Mulvihill N, et al. Helicobacter pylori serology in patients with angiographically documented coronary artery disease. Am $\mathcal{f}$ Cardiol 1999;83:1664-6.

4 Danesh J. Is there a link between chronic helicobacter pylori infection and coronary heart disease? Eur $\mathcal{F}$ Surg 1998; suppl 582:27-31.

5 Danesh, J, Wong Y, Ward M, et al. Chronic infection with helicobacter pylori, chlamydia pneumoniae, or helicobacter pylori, chlamydia pneumoniae, or
cytomegalovirus: population based study of coronary heart

6 Zhou YF, Leon M, Waclawiw MA, et al. Association Zhou YF, Leon M, Waclawiw MA, et al. Association
between prior cytomegalovirus infection and the risk of restenosis after coronary atherectomy. N Engl F Med 1996; 335:624-30. 
7 Manegold C, Alwazzeh M, Jablonovski $\mathrm{H}$, et al. Prior cytomegalovirus infection and the risk of restenosis after cytomegalovirus infection and the risk of restenosis after
percutaneous transluminal coronary balloon angioplasty. percutaneous transluminal
Circulation 1999;99:1290-4.

8 Blum A, Giladi M, Weinberg M, et al. High anti cytomegalovirus $\mathrm{IgG}$ antibody titer is associated with coronary disease and may predict post coronary balloon angioplasty restenosis. Am 7 Cardiol 1998;81:866-8.

9 Carlsson J, Miketic S, Mueller KH, et al. Previous cytomegalovirus or chlamydia pneumoniae infection and risk of restenosis after percutaneous transluminal coronary angioplasty. Lancet 1997;350:1225.

10 Tiran A, Tio R, Ossewaarde J, et al. Coronary angioplasty induces rise in chlamydia pneumoniae specific antibodies. $\mathcal{F}$ Clin Microbiol 1999;37:1013-17.

11 Oflaz H, Nisanci P, Cekmeceli P, et al. Chlamydia pneumoniae antibodies and their relationship with restenosis in coronary heart disease patients [abstract]. Eur Heart $\mathcal{f}$ 1999;20 (abstract suppl):521.

12 Zhou Y, Csako G, Grayston T, et al. Lack of association of restenosis following coronary angioplasty with elevated
C-reactive protein levels or seropositivity to Chlamydiae C-reactive protein levels or seropositivity
pneumoniae. Am f Cardiol 1999;84:595-7.

13 Hoffmann R, Mintz GS, Dussaillant GR, et al. Patterns and Hoffmann R, Mintz GS, Dussaillant GR, et al. Patterns and
mechanisms of in-stent restenosis. a serial intravascular ultrasound study. Circulation 1996;94:1247-54.

14 Bertrand M, Bauters C. Cytomegalovirus infection and coronary restenosis. Circulation 1999;99:1278-9

15 Mehran R, Mintz G, Hong M, et al. Validation of the in vivo intravascular neointimal hyperplasia volumes. $\mathcal{F} \mathrm{Am} \mathrm{Coll}$ Cardiol 1998;32:794-9.

16 Speir E, Modali R, Huang ES, et al. Potential role of human cytomegalovirus and $\mathrm{p} 53$ interaction in coronary restenosis. Science 1994;265:391-4.

17 Libby P, Egan D, Skarlatos S. Roles of infectious agents in atherosclerosis and restenosis. An assessment of the evidence and need for future research. Circulation 1997;96: 4095-103.

18 Epstein SE, Speir E, Zhou Y, et al. The role of infection in restenosis and atherosclerosis: focus on cytomegalovirus. Lancet 1996;348:13-17.

19 Danesh J, Collins R, Peto R. Chronic infection and coronary heart disease: is there a link? Lancet 1997;350:430-6.

20 Kimura T, Kaburagi S, Tamura T. Remodelling of human coronary arteries undergoing coronary angioplasty or atherectomy. Circulation 1997;96:475-83.

21 Anderson $\mathrm{H}$, Maeng $\mathrm{M}$, Thorwest $\mathrm{M}$, et al. remodelling rather than neointimal formation explains lumen narrowing after deep vessel wall injury: insights from a porcine coronary (re)stenosis

22 Mintz GS, Popma J, Pichard A. Arterial remodelling after coronary angioplasty: a serial intravascular ultrasound study. Circulation 1996;94:35-43.

23 Hoffmann R, Mintz G, Pichard A, et al. Intimal hyperplasia thickness at follow-up is independent of stent size: a serial intravascular ultrasound study. Am 7 Cardiol 1998;82: 1168-72.

24 Limpijankit T, Waksman R, Yock P, et al. Intravascular ultrasound volumetric assessment of intimal hyperplasia in stent treated with intracoronary radiation. Am $\mathcal{F}$ Cardiol 1999;84:850-4.

25 Smith K, Parsonnet J. Association between prior cytomegalovirus infection and the risk of restenosis after coronary atherectomy [letter]. N Engl F Med 1997;336:587.

26 Serruys P. Is there MUSIC in IVUS guided stenting? Is this MUSIC going to be a MUST? Eur Heart F 1998;19:1122-4.

27 Kastrati A, Schömig A, Elezi S, et al. Predictive factors of restenosis after coronary stent placement. $\mathcal{F} \mathrm{Am}$ Coll Cardiol 1997;30:1428-36.

\section{IMAGES IN CARDIOLOGY}

\section{Magnetic resonance imaging of cardiac osteosarcoma}

A 30 year old woman was admitted to our hospital because of shortness of breath and general fatigue that developed progressively. Transthoracic and transoesophageal echocardiography demonstrated an abnormal mass located just proximal to the pulmonary valve and occupying the right ventricular outflow tract. The magnetic resonance images in transverse (left) and sagittal (right) views also showed a huge mass of heterogenous density in the right ventricular outflow tract. A moderate amount of pericardial effusion was noted. The increase in size of the right ventricle with presence of the flattened ventricular septum suggested increased right ventricular pressure. In the sagittal view (right) part of the mass seemed to invade the ventricular free wall extensively, suggesting the possibility of a malig-

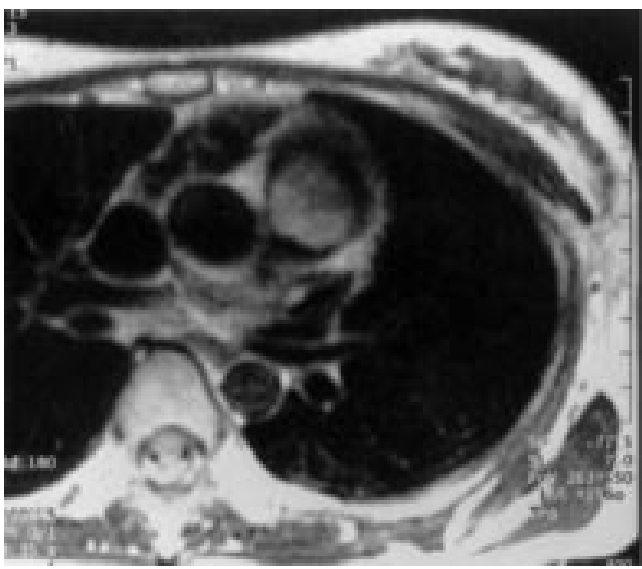

nant tumour. As a result, the right ventricular outflow tract and part of the pulmonary artery was extensively resected and reconstructed. Histology from the resected mass confirmed that the tumour consisted of an osteosarcoma that commonly occurs in the left atrium and frequently demonstrates calcification, although the magnetic resonance image did not show the calcified lesion. Magnetic resonance imaging can help to differentiate malignant from benign tumours by revealing certain features - such as a broad base of attachment as shown in this case-probably better than conventional echocardiography.

MASAKAZU YAMAGISHI NAOAKI YAMADA SACHIO KURIBAYASHI myamagi@hsp.ncvc.go.jp

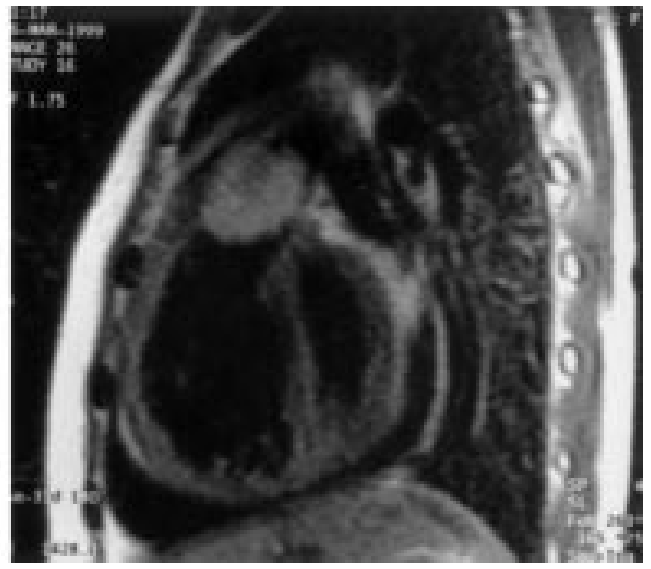

